\title{
Crystal structure of polymeric carbon nitride and determination of its process-temperature-induced modifications
}

\author{
T. Tyborski, ${ }^{1,2}$ C. Merschjann, ${ }^{1,3}$ S. Orthmann, ${ }^{1}$ F. Yang, ${ }^{1}$ M.-Ch. Lux-Steiner, ${ }^{1}$ and Th. Schedel-Niedrig ${ }^{1}$ \\ ${ }^{1)}$ Helmholtz-Zentrum Berlin für Materialien und Energie GmbH, D-14109 Berlin, \\ Germany \\ ${ }^{2)}$ Max-Born-Institut für Nichtlineare Optik und Kurzzeitspektroskopie, D-12489 Berlin, \\ Germany ${ }^{\text {a) }}$ \\ ${ }^{3)}$ Universität Rostock, D-18055 Rostock, Germany
}

Based on the arrangement of two-dimensional melon, we construct a unit cell for polymeric carbon nitride $(\mathrm{PCN})$ synthesized via thermal polycondensation whose theoretical diffraction powder pattern includes all major features measured in X-ray diffraction. With the help of that unit cell, we describe the processtemperature-induced crystallographic changes in PCN that occur within a temperature interval between $510^{\circ} \mathrm{C}$ and $610^{\circ} \mathrm{C}$. We also discuss further potential modifications of the unit cell for PCN. It is found that both triazine and heptazine-based $\mathrm{g}-\mathrm{C}_{3} \mathrm{~N}_{4}$ can only account for minor phases within the investigated synthesis products.

Keywords: polymeric carbon nitride, $\mathrm{g}-\mathrm{C}_{3} \mathrm{~N}_{4}$, thermal polycondensation, crystal structure

Polymeric carbon nitride (PCN) stands for a very remarkable class of materials. It reveals photocatalytic activity, both as pristine powder and as a nanocrystalline film in a heterojunction photocathode. ${ }^{1-3}$ Furthermore, PCN only consists of abundant elements, can be synthesized via a straightforward, scalable process and offers a high chemical and thermal stability. ${ }^{4}$ Light-induced hydrogen release on the basis of PCN in an acidic aqueous solution under the presence of an electron donor has been demonstrated. ${ }^{1}$ At the same time, a synthesis-parameter and thus crystal structure dependence of the photocatalytic activity as well as of the photophysics of PCN is documented. ${ }^{1,4-6}$ These features combined in PCN establish a far-ranging interest on that class of materials, especially with respect to sustainable hydrogen production. ${ }^{7}$ The synthesis of PCN via a bulk thermal polycondensation is characterized by both the reaction-temperaturedependent hydrogen content and the degree of polymerization within a temperature range of about $510^{\circ} \mathrm{C}$ up to $610^{\circ} \mathrm{C} \cdot{ }^{4,8-10}$ The thermal polycondensation itself is a continuous process that reveals several dominant phases at certain temperatures, such as melamine at $250^{\circ} \mathrm{C}$ or melem at $450^{\circ} \mathrm{C} .{ }^{4,9}$ While some of these dominant phases can be examined from a crystallographic point of view, the crystallographic structure of PCN has only been described insufficiently. ${ }^{11} \mathrm{X}$-ray diffraction (XRD) data of PCN reveal preferentially two major, slightly reactiontemperature-dependent reflexes at $2 \Theta \approx 27.3^{\circ}(3.26 \AA)$ and $2 \Theta \approx 13.2^{\circ}(6.70 \AA)$. These reflexes are understood as diffraction at graphite-like sheets for the former and as diffraction at a periodic structural feature within the sheets for the latter diffraction angle. ${ }^{4}$ The graphite-like sheets exhibit pores and consist of polymerized heptazine units. ${ }^{4,12,13}$

However, so far a distinct unit cell with a concrete space group for $\mathrm{PCN}$ that combines the listed structural aspects and is in good agreement with the measured XRD powder patterns has not been identified.

The usual way to determine the crystal structure of a certain material on the basis of its powder pattern is executed as follows. ${ }^{14-16}$ (1) The unit cell is determined by indexing the peaks of the powder pattern. (2) The powder pattern is decomposed into integrated intensities $I_{h, k, l}$. (3) The space group is assigned based on the systematic absence of several $I_{h, k, l}$. (4) The phase problem is solved, e.g. with the help of the Patterson Method. (5) The structure is refined, typically with Rietveld refinement.

This proper structure analysis assumes a high purity as well as a high crystallinity of the investigated material which in the case of PCN synthesized via thermal polycondensation can both not be afforded. ${ }^{2,4}$ This is the point where subjective judgements and feasible arguments come to the fore in order to overcome the steps (1)-(3) while a proper refinement does not stand a chance. In this work, we contrast melon-based unit cells with triazine - and heptazine-based unit cells and compare their theoretical X-ray diffraction patterns with measured patterns from PCN. Our findings of that analysis suggest that the major phase of $\mathrm{PCN}$ is based on melon sheets as well as that $\mathrm{g}-\mathrm{C}_{3} \mathrm{~N}_{4}$ can only account for a minor phase within the investigated synthesis products. We also discuss geometrical modifications of the melon-based PCN unit cell and demonstrate their influence on X-ray diffraction patterns. Furthermore, we use the melon-based PCN unit cell in order to describe crystallographic changes within PCN that come along with higher process temperatures. Details concerning the crystallographic structures can be found in the supporting information.

a) Electronic mail: tybo@physik.fu-berlin.de 


\section{A. EXPERIMENTAL SECTION}

\section{Synthesis of PCN}

Dicyandiamide (Aldrich, $99 \%$, about $2.5 \mathrm{~g}$ ) was heated to temperatures between $490^{\circ} \mathrm{C}$ and $610^{\circ} \mathrm{C}$ (in $20^{\circ}$-steps) at a rate of $25^{\circ} \mathrm{min}^{-1}$ in a ceramic crucible within a muffle furnace under continuous $\mathrm{N}_{2}$ gas flow. In a first processing step, the thermal treatment lasts for five minutes after which the resulting products were grounded. In a second processing step, the grounded materials were heated to the respective processing temperatures for another three hours under $\mathrm{N}_{2}$ gas flow, after what they were allowed to cool down passively in the furnace.

\section{Structural characterization}

Structural phase analysis of the PCN powders was performed by X-ray diffractometry (Bruker AXS D8, $\mathrm{Cu}_{\mathrm{K}_{\alpha}}$ line) in a $\Theta-2 \Theta$ configuration. Approximately $100 \mathrm{mg}$ of each PCN powder was analyzed for one hour between $2 \Theta=10^{\circ}$ and $2 \Theta=90^{\circ}$. The resulting powder patterns have been backround-corrected with an exponential function in order to minimize the influence of diffuse scattering. Since they do not exhibit narrow reflexes, the powder patterns have been smoothed for the purpose of emphasizing their features with respect to the noise.

\section{Structure analysis}

Each discussed theoretical unit cell was analyzed in a numerical X-ray diffraction experiment. These analysis were performed with the program Diamond 3.2 $i$ and reviewed with the program PowderCell 2.4. ${ }^{17-20}$ The following parameters (adjustable in the software) were used: $\mathrm{X}$-ray laboratory source with wavelength $\lambda=1.54059 \AA$, $2 \Theta_{\min }=10^{\circ}$ and $2 \Theta_{\max }=90^{\circ}$, activated Lorentz factor, activated Polarization factor and disabled displacement factor.

Depending on the particular analyzed unit cell, the software calculates the corresponding structure factors and thus the corresponding diffraction pattern. The physical background of that calculation is the independent-atom model (IAM), in which the electronic charge density is exclusively located at the atoms.

All discussed numerical values for reflex positions or separation distances such as $d_{\mathrm{s}}$ or $d_{\mathrm{c}}$ have been measured with the program Diamond 3.2 $i$ on the basis of the corresponding atomic positions and crystal parameters. The mean separation distance $d_{\mathrm{c}}$ is defined as $d_{\mathrm{c}}=\frac{1}{5} \sum_{i=1}^{5} d_{\mathrm{c}_{i}}$ in which $d_{\mathrm{c}_{i}}$ are the separation distances of the (five) particular pairs of nitrogen atoms that are located face to face with each other along the heptazine chains (figure $5)$.

\section{B. Graphitic carbon nitride $\mathbf{g}-\mathrm{C}_{3} \mathbf{N}_{4}$}

A triazine-based structure for $\mathrm{g}-\mathrm{C}_{3} \mathrm{~N}_{4}$ was theoretically suggested by Teter and Hemley in $1996 .{ }^{12}$ Next to the unit cell (upper inset), figure 1 shows the theoretical diffraction pattern of that material in combination with a measured XRD pattern of $\mathrm{PCN}$. This comparison reveals fundamental structural differences between PCN and triazine-based $\mathrm{g}-\mathrm{C}_{3} \mathrm{~N}_{4}$. Especially the intense reflexes of PCN at diffraction angles smaller than $2 \Theta=20^{\circ}$ cannot be attributed to g- $\mathrm{C}_{3} \mathrm{~N}_{4}$. Similarities can be found for the $\mathrm{g}-\mathrm{C}_{3} \mathrm{~N}_{4}$-reflexes $(011)$ at $2 \Theta=25.40^{\circ},(002)$ at $2 \Theta=26.50^{\circ}$ and $(020)$ at $2 \Theta=44.07^{\circ}$.

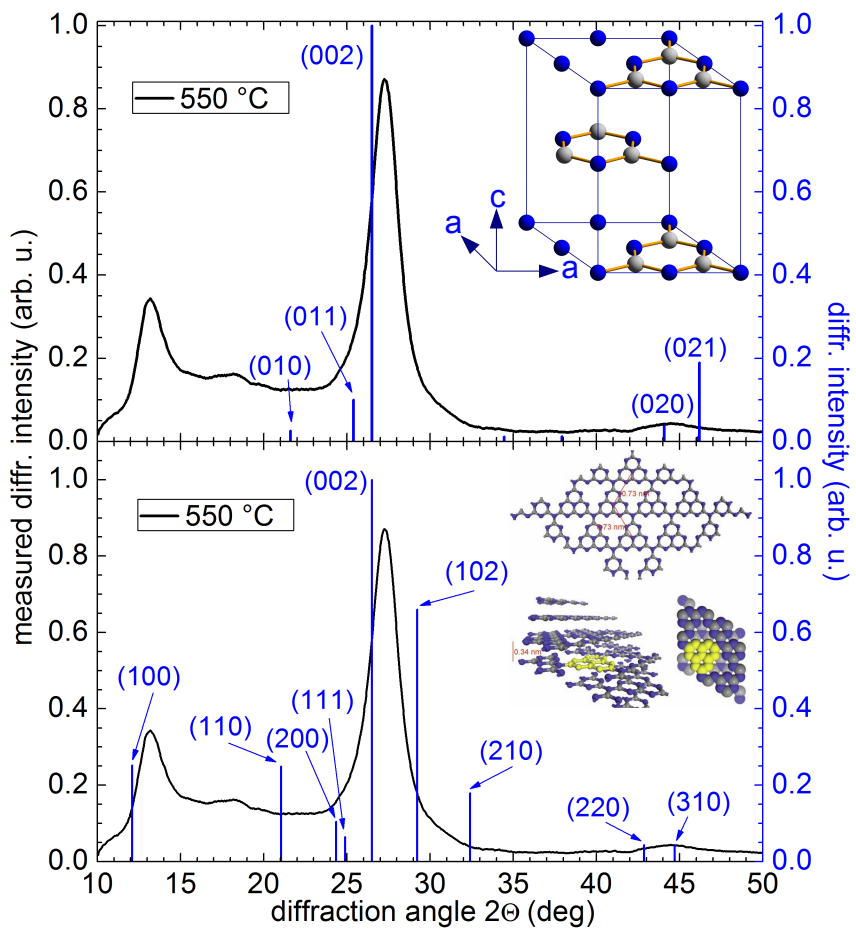

FIG. 1. Top: Unit cell for triazine-based $\mathrm{g}-\mathrm{C}_{3} \mathrm{~N}_{4}$ with space group $P \overline{6} m 2$ (187), $a=4.742 \AA, c=6.7205 \AA$ and 14 atoms $(8 \mathrm{~N}$ (blue $)+6 \mathrm{C}($ grey $))$ together with the diffraction pattern of PCN (black curve) and the most intense diffraction reflexes of an ideal triazine-based $\mathrm{g}-\mathrm{C}_{3} \mathrm{~N}_{4}$-crystal (blue lines). ${ }^{12}$ Bottom: Structure of heptazine-based g- $\mathrm{C}_{3} \mathrm{~N}_{4}$ synthezised by Bojdys et al. with space group $P \overline{6}_{3} \mathrm{~cm}$ (185), $a=8.434 \AA, c=6.722 \AA$ and its most intense diffraction reflexes (grey lines). ${ }^{21}$

Bojdys et al. have demonstrated an ionothermal synthesis route for a substance they also call $\mathrm{g}-\mathrm{C}_{3} \mathrm{~N}_{4} \cdot{ }^{21}$ Their material (lower inset figure 1) exhibits the largest crystallites known so far for any graphite-like carbon nitride, showing diameters of about $200 \mathrm{~nm}$. The corresponding structure analysis reveals the hexagonal space group $\mathrm{P}_{3} \mathrm{~cm}$ (185). However, XRD analysis also indicates that this is a diverse material compared to both the triazinebased $\mathrm{g}-\mathrm{C}_{3} \mathrm{~N}_{4}$ and the PCN synthesized via thermal polycondensation which necessitates the consideration of a different unit cell for PCN.

\section{Unit cell for PCN}

We construct a unit cell for $\mathrm{PCN}$ on the basis of poly(aminoimino)heptazine $\left[\mathrm{C}_{6} \mathrm{~N}_{7}\left(\mathrm{NH}_{2}\right)(\mathrm{NH})\right]_{\mathrm{n}}$, also known as "melon", whose structure has been analyzed by 
Wang et al. and Lotsch et al. ${ }^{1,10}$ The relative positions of the atoms within the melon sheet were extracted digitally from figure 12 in Ref. 10 with the aid of the image data processing program ImageJ $1.43 u{ }^{10,22,23}$ In this procedure, the central points of the illustrated grey and black dots served as the atomic coordinates for nitrogen and carbon. We added coordinates for the missing hydrogen atoms. With these atomic coordinates, we construct a theoretical unit cell for PCN. The relative alignment of the atoms in the unit cell can be modified mathematically such that, for example, a buckling or different stacking motifs can be introduced. Since the graphical melon representation in Ref. 10 and the extraction of the atomic coordinates are inherently correlated with minor uncertainties, we initially assume the lowest possible symmetry (space group $P 1$ ) in our unit cell. The cell parameters have been chosen to be in line with the measured XRD data of our PCN samples as well as with the findings from Lotsch et al. ${ }^{10}$

In this way, we suggest a unit cell for PCN synthesized via thermal polycondensation of dicyandiamide with the following parameters: space group $P 1$, $a=16.2 \AA, b=12.1 \AA, c=3.275 \AA$ and $\alpha=\beta=\gamma=90^{\circ}$, in which $c$ and $\gamma$ exhibit a process-temperature dependence (figure 4 and figure 5).
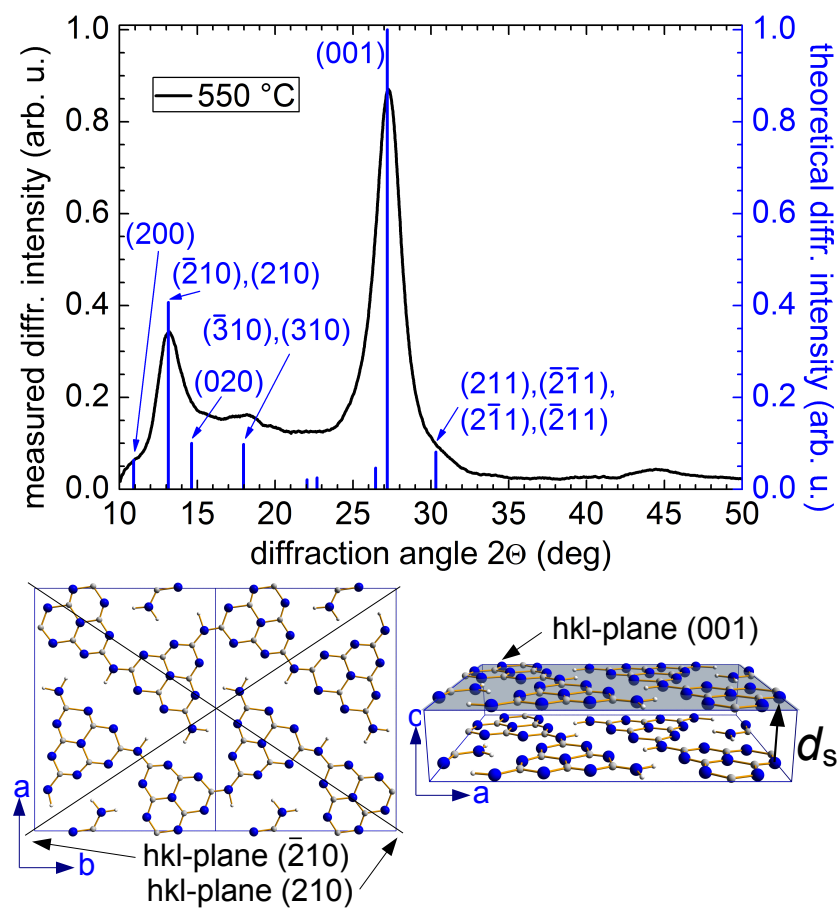

FIG. 2. Triclinic unit cell for PCN with flat melon sheets, an A-A-stacking motif and 72 atoms $(36 \mathrm{~N}+24 \mathrm{C}+12 \mathrm{H})$. Bottom: PCN lattices along c-direction and with view into the stacked melon sheets, each with highlighted hkl-planes. Top: Measured XRD pattern (black curve) and the most intense diffraction reflexes of the corresponding ideal PCN (blue lines).
Figure 2 shows a unit cell for A-A-stacked PCN with flat melon sheets. The corresponding theoretical diffraction pattern is opposed to a measured powder pattern of PCN in the upper part of figure 2. This comparison reveals that especially the strong reflexes $(\overline{2} 10),(210)$ at $2 \Theta=$ $13.14^{\circ}$ and $(001)$ at $2 \Theta=27.21^{\circ}$ match the measured powder pattern with respect to their angular positions and intensities persuasively. The evident peak between the major reflexes can be understood as diffraction at the hkl-planes $(\overline{3} 10)$ and $(310)$ at $2 \Theta=17.97^{\circ}$. The pairs $(\overline{2} 10),(210)$ and $(\overline{3} 10),(310)$ are each overlapping reflexes.

Three modifications need to be discussed in the further procedure compared to flat A-A stacked PCN: buckling within singular melon sheets, different stacking motifs and the influence of the process temperature. ${ }^{4}$

\section{Buckling}

Compared to infinite $\mathrm{C}_{6} \mathrm{~N}_{8}$ sheets, in which the energetic advantage of a sinusoidal buckling with an amplitude of $0.7 \AA$ parallel to the plane normal is calculated to be $11 \mathrm{~kJ} \mathrm{~mol}^{-1}$ per neighboring $\mathrm{N}-\mathrm{N}$ pair, the melon sheets in PCN very likely feature a related buckling., ${ }^{4,24}$

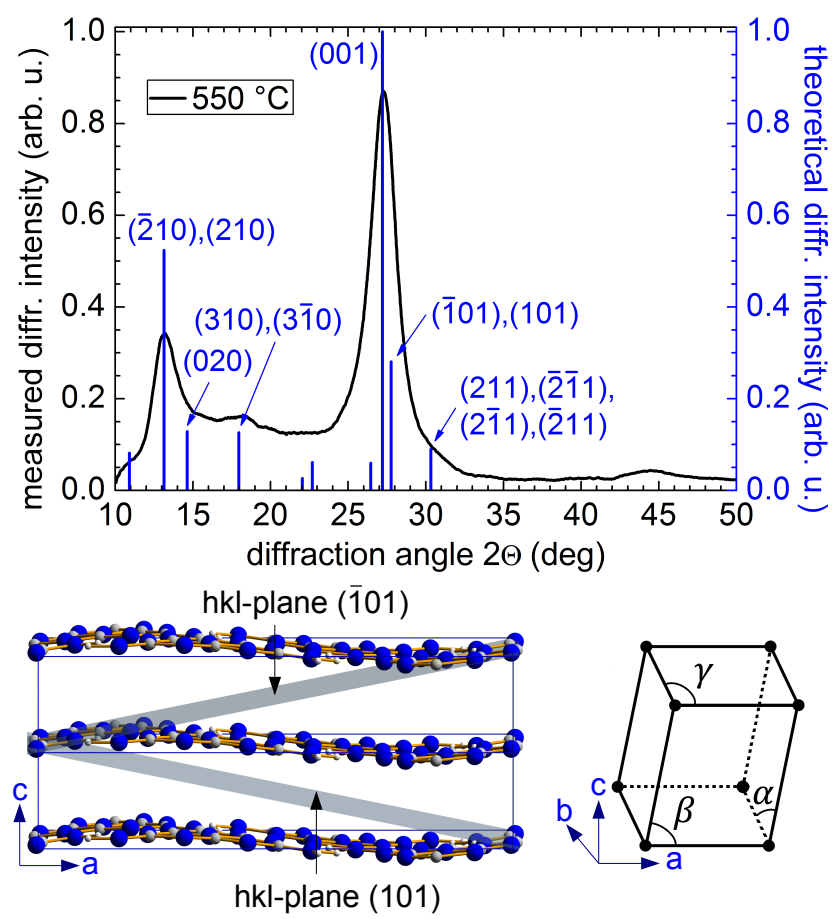

FIG. 3. Triclinic unit cell for PCN with sinusoidally buckled melon sheets (parallel to $a$-c-plane, $0.7 \AA$ amplitude) and an A-A-stacking motif. Bottom: PCN lattice with view into the stacked melon sheets, highlighted hkl planes and a triclinic unit cell. Top: Measured XRD pattern (black curve) together with the most intense theoretical diffraction reflexes of the corresponding PCN lattice (blue lines).

In the special case of a sinusoidal buckling parallel to the $a-c$-plane, as demonstrated in figure 3 , associated features in the diffraction pattern are arising reflexes ( $\overline{1} 01)$ 
and $(101)$, both at $2 \Theta=27.77^{\circ}$. Emerging reflexes $(0 \overline{1} 1)$ and $(011)$, at $2 \Theta=28.21^{\circ}$, are the result of a sinusoidal buckling parallel to the $b$ - $c$-plane (not shown). A sinusoidal buckling parallel to the c-direction and along the $a$ - $b$-diagonal causes weak emerging $(\overline{1} 01),(101)$ - and $(0 \overline{1} 1),(011)$ - as well as $(111),(\overline{1} 11),(1 \overline{1} 1),(\overline{1} 11)$ - reflexes (not shown). The corresponding buckling-induced increase of N-N-distances between nearest neighbors in one heptazine unit accounts for roughly $5 \times 10^{-3} \AA$, the distance $d_{c}$ (figure 5 ) between the in-plane heptazine chains increases by about $10 \times 10^{-3} \AA$ while the intraplanar N$\mathrm{N}$-distances of second nearest neighbors extend by about $2 \times 10^{-3} \AA$. Comparable, minor changes in the theoretical diffration patterns can be observed for slightly varied buckling amplitudes.

\section{Stacking motifs}

If one assumes the stacking of the melon sheets in PCN to be of the form A-B, the (001)-reflex is replaced by a (002)-reflex in the corresponding diffraction pattern and the amount of atoms per unit cell is doubled, generally. A displacement of every second melon sheet $0.5 \times a$ along the a-axis leads to a diffraction pattern that features forbidden reflexes $(\overline{3} 10)$ and (310) only (not shown). In exchange a relocated and enhanced quad reflex $(311),(\overline{3} 11),(3 \overline{1} 1),(\overline{3} \overline{1} 1)$ appears at $2 \Theta=22.55^{\circ}$ (not shown). In the case of a displacement $0.5 \times b$ along the b-axis, the reflexes $(\overline{2} 10)$ and $(210)$ vanish whereas a strong quad reflex $(211),(\overline{2} 11),(2 \overline{1} 1),(\overline{2} \overline{1} 1)$ emerges at $2 \Theta=18.89^{\circ}$ (not shown). A combination of these two displacements $(0.5 \times a+0.5 \times b)$ also leads to vanishing $(2 \overline{1} 0),(210)$ - and arising $(211),(\overline{2} 11),(2 \overline{1} 1),(\overline{2} \overline{1} 1)$ reflexes (not shown).

\section{Process-temperature-induced influence on the crystal structure of PCN}

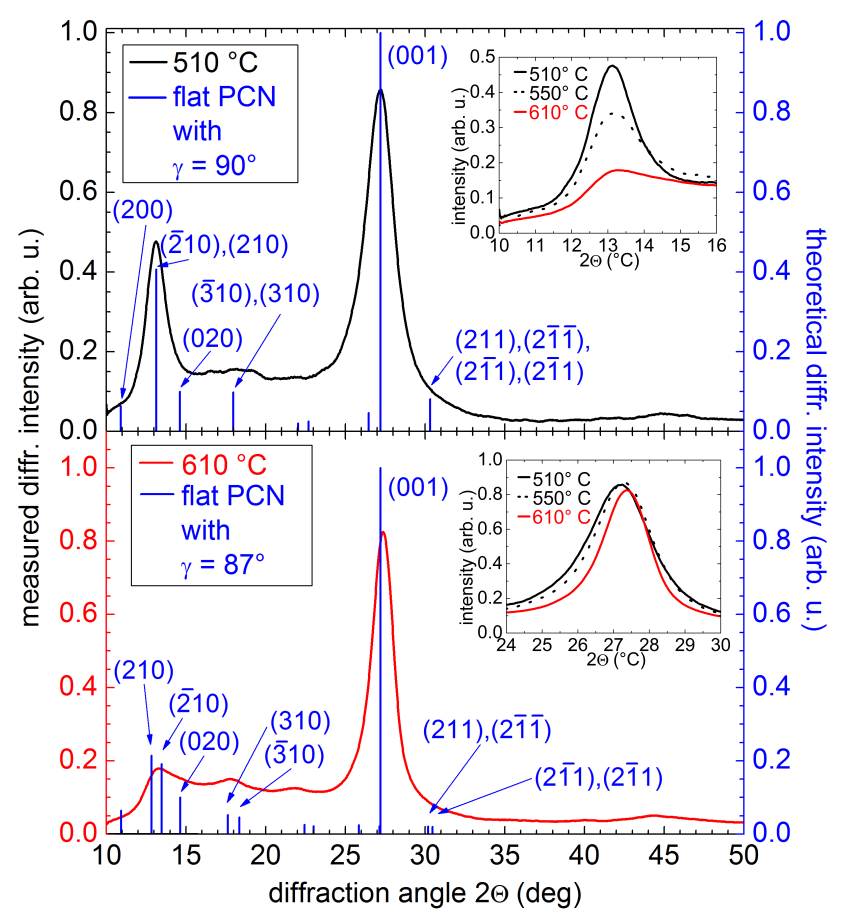

FIG. 4. Measured XRD patterns of PCN synthesized at $510{ }^{\circ} \mathrm{C}$ (top) and at $610{ }^{\circ} \mathrm{C}$ (bottom), together with the most intense theoretical reflexes of flat, A-A-stacked PCN with two different unit cell angles $\gamma$. Figure insets show an enlargement of the peaks at $2 \Theta=13.2^{\circ}$ and $2 \Theta=27.2^{\circ}$, respectively, for three different process temperatures.

Increasing the process temperature of PCN leads to significant changes in the measured diffraction patterns. ${ }^{4,21}$ As can be seen in figure 4 , the higher the process temperature, the weaker and broader is the peak at $2 \Theta=13.2^{\circ}$. At the same time the peak at $2 \Theta=27.2^{\circ}$ is shifted towards larger diffraction angles. While the weakening and broadening of the $2 \Theta=13.2^{\circ}$-peak is clearly observable, one can only observe a slight shift towards higher diffraction angles for the $2 \Theta=27.2^{\circ}$-peak. Next to the specified changes, a decreasing FWHM of the $27.2^{\circ}$-peak from roughly $1.6^{\circ}$ to $1.3^{\circ}$ is observable.

These process-temperature-induced changes can be understood on the basis of the triclinic PCN suggestion. During the thermal polycondensation, the heptazine units begin to chemically condensate at temperatures of around $510^{\circ} \mathrm{C}$, thus forming PCN. Further heating to temperatures of around $610^{\circ} \mathrm{C}$ reduces the hydrogen content via ammonia release, pointing to an enhanced crystallinity. ${ }^{4}$ An enhanced crystallinity along the c-axis is clearly observable in the shift of the $27.2^{\circ}$-peak and its reduced FWHM, indicating an increased number of stacked melon sheets per crystallite as well as a reduced stacking distance $d_{\mathrm{s}}$ (figure 2). Necessarily correlated to the decreasing distance between the sheets is the decreasing distance of particular nitrogen atoms. Since all nitrogen atoms in PCN exhibit an unbound pair of electrons, large N-N-distances are energetically beneficial. So one 
single melon sheet distorts its two-dimensional ordering such, that the inner nitrogen distances increase. For that reason, the angle $\gamma$ of PCN's triclinic unit cell is slightly shifted to values $<90^{\circ}$, as shown in figure 5 .

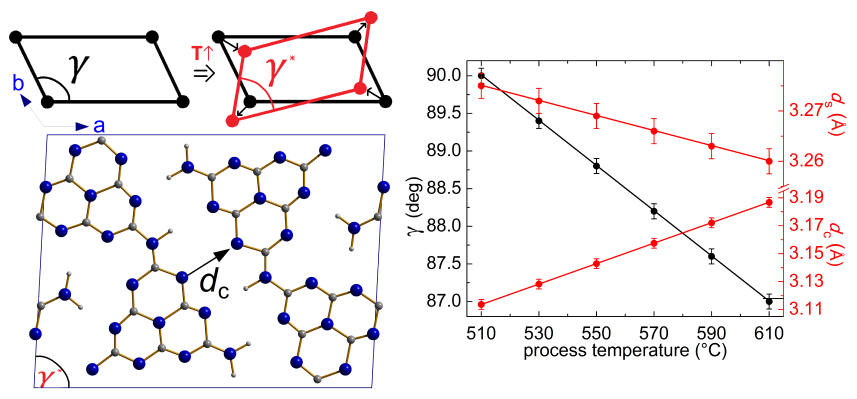

FIG. 5. Left: Scheme of the temperature-induced distortion of PCN's crystal structure together with a section of a distorted unit cell $\left(\gamma^{*}=87^{\circ}\right)$ and the mean separation distance between two heptazine chains $d_{\mathrm{c}}$. Note that the scheme is illustrated excessively for an enhanced clarity. Right: Relation between $\gamma, d_{\mathrm{c}}, d_{\mathrm{s}}$ and the process temperature (in flat, A-A-stacked PCN).

While the stacking distance $d_{\mathrm{s}}$ stays unchanged with respect to a decreasing angle $\gamma$, the heptazine units are slightly distorted and the mean separation distance $d_{\mathrm{c}}$ between the heptazine chains is increased from $3.11 \AA$ to $3.19 \AA$ with a slope of $7 \times 10^{-4} \AA /{ }^{\circ} \mathrm{C}$. This distortion influences the theoretical diffraction pattern of PCN such, that the overlapping reflexes $(\overline{2} 10),(210)$ and $(\overline{3} 10),(310)$ are displaced against each other (figure 4). A very similar behavior can be observed for an enlarged angle $\gamma$. But enlarging $\gamma$ is correlated to decreased distances $d_{\mathrm{c}}$, which makes this kind of distortion unplausible. Slightly varying the angles $\alpha$ and $\beta$ ends up in only minorly changed theoretical diffraction patterns. The discussed results can be found for the flat as well as for buckled PCN.

\section{DISCUSSION}

As mentioned before, the applied independent-atom model restricts the electron density at the atoms. Thus, the electron density is described by the spherically averaged density of the isolated atoms. ${ }^{25}$ The model works very convincingly for solely heavy atoms in the unit cell. This is because in that case, the valence shells are only a minor part of the total electron density at which the $\mathrm{X}$-ray photons are scattered. It affords unaccuracies for light atoms. For example, the bond length of a covalent $\mathrm{C}-\mathrm{H}$ bond is typically underestimated within the IAM picture since the hydrogens electron density is significantly distributed over the bond. ${ }^{25,26}$ Nevertheless, the IAM has been the basis of X-ray structure analysis since its inception. ${ }^{25}$ Especially for PCN, whose X-ray diffraction patterns do not allow an advanced structure analysis, the IAM offers an established tool in order to determine a unit cell for PCN.

We have also analyzed the influence of atomic displace- ment factors on the diffraction patterns of PCN. For that purpose, we have used a unit cell for flat, A-A-stacked PCN and implemented an isotropic displacement factor of $0.04 \AA^{2}$ for each atom, according to the melem crystal investigated by Jürgens et al. and A. Sattler et al. ${ }^{9,11}$ The corresponding findings are slightly varied total reflex intensities and intensity ratios. For example, the (001)reflex intensity is decreased by about $15 \%$ and the sum intensity of $(210)$ and $(\overline{2} 10)$ by about $4 \%$. This results in a modified intensity ratio of $(\mathrm{I}[(210)]+\mathrm{I}[(\overline{2} 10)]) / \mathrm{I}[(001)]$ from approximately 0.41 for disabled displacement factors (figure 2 ) to $\approx 0.46$ for enabled displacement factors (not shown).

The proposed unit cell for PCN and its modifications do not allow inversion symmetry. However, Lotsch et al. describe melons in-plane symmetry with the plane group $p 2 g g$, which includes 2-fold rotations, glide-reflections and translations. ${ }^{10}$ In order to conserve the 2-fold rotational symmetry within the melon sheets and thus inversion symmetry within the PCN unit cells, the following procedure may be applied: One could construct a PCN unit cell with the discussed cell parameters, but only include half of the atoms per unit cell, such that an inversion maps the missing atoms to the appropriate atomic positions. The corresponding space group, which allows this symmetry operation, is the space group $P \overline{1}$. The theoretical diffraction patterns for inversionsymmetric PCN (space group $P \overline{1}$ ) are very similar to those of PCN without inversion symmetry (space group $P 1)$. For example, the sum intensity of the (210) and (210)-reflex is decreased by about $4 \%$ for an inversion symmetry in flat, A-A-stacked PCN, compared to the non-inversion-symmetric case, while the diffraction angle stays unchanged.

We propose a triclinic crystal lattice for $\mathrm{PCN}$, since this is the only crystal system for which $a \neq b \neq c$ and $\gamma \neq 90^{\circ}$ which is necessary for the description of the discussed process-temperature-induced modifications in PCN. Varying $\alpha$ and $\beta$ leads to shifted (001) - and $(210),(\overline{2} 10)$-reflexes in the diffraction patterns towards higher diffraction angles and modified intensities. For $\alpha=85^{\circ}$ or $\alpha=95^{\circ}$ and untouched residual lattice parameters, the (210) - and ( $\overline{2} 10)$-reflexes are displaced by about $\Delta 2 \Theta=0.02^{\circ}$, whereas the (001)-reflex is displaced by about $\Delta 2 \Theta=0.11^{\circ}$. For $\beta=85^{\circ}$ or $\beta=95^{\circ}$ the (210) - and (210)-reflexes are displaced by about $\Delta 2 \Theta=0.03^{\circ}$, whereas the (001)-reflex is displaced by about $\Delta 2 \Theta=0.11^{\circ}$.

Seyfarth et al. have discussed some possible arrangements of stacked melon sheets based on theoretical calculations. Their findings lead to significant relative displacements. ${ }^{27}$ Implementing these displacements into the unit cell for flat PCN results in theoretical diffraction patterns that are all not sufficient to reconstruct the measured ones (see supporting information).

The only traceable hint for graphitic carbon nitride g$\mathrm{C}_{3} \mathrm{~N}_{4}$ within the synthesized PCN is the weak but omnipresent peak around $2 \Theta=44^{\circ}$ in the measured diffrac- 
tion patterns (figure 1). This one can be related to the (200)-reflex of triazine-based $\mathrm{g}-\mathrm{C}_{3} \mathrm{~N}_{4}$ or to the (300)reflex of heptazine-based $\mathrm{g}-\mathrm{C}_{3} \mathrm{~N}_{4}$ (appendix). Thus, inor case, $\mathrm{g}-\mathrm{C}_{3} \mathrm{~N}_{4}$ is only a minor outcome of the thermal polycondensation of dicyandiamide. This is also consistent with the reported hydrogen content of $1-2 \mathrm{wt} \%$ in the synthesized PCN, since the theoretical value for its hydrogen content is expected to be $1.27 \mathrm{wt} \% .^{4,21}$

One finds synthesis products for process temperatures below $510^{\circ} \mathrm{C}$ or above $610^{\circ} \mathrm{C}$ whose measured diffraction patters deviate significantly from the ones of PCN. . $^{4,5,21}$ This is mainly caused by a considerable amount of nonpolymerized melem within the synthesis products for process temperatures below $510{ }^{\circ} \mathrm{C}$ and a degradation of PCN for process temperatures above $610^{\circ} \mathrm{C} \cdot{ }^{4,9,11}$ The process-temperature dependence of the photophysics of PCN can be extended to temperatures from about $400^{\circ} \mathrm{C}$ to $610^{\circ} \mathrm{C}$. The reason for that is the building unit $\mathrm{C}_{6} \mathrm{~N}_{10} \mathrm{H}_{\mathrm{x}}$, "melem" in the non-polymerized and "heptazine" in the polymerized state, which is omnipresent in that temperature range and in which the photophysics is found to take place. ${ }^{6,9}$ These units are more densely packed for higher process temperatures, thus enhancing the electronic interaction between them which influences PCN's optical properties. ${ }^{6}$

Furthermore, we suggest the space group $P \overline{6}(174)$ for heptazine-based $\mathrm{g}_{-} \mathrm{C}_{3} \mathrm{~N}_{4}$. From a crystallographic point of view, this is the only hexagonal space group that includes the symmetry operations for which the sum of Wyckoff multiplicities is identical to the generated atoms per heptazine-based $\mathrm{g}_{-} \mathrm{C}_{3} \mathrm{~N}_{4}$ unit cell. A-A-stacked as well as A-B-stacked heptazine-based $\mathrm{g}-\mathrm{C}_{3} \mathrm{~N}_{4}$ is thinkable (see supporting information).

To conclude our analysis about the crystal structure of PCN and the associated discussion, we sum up our findings as follows. We expect PCN to mainly consist of

- buckled, A-A-stacked PCN and/or

- flat, A-A-stacked PCN

with process-temperature-dependent unit cell parameters $c$ and $\gamma$. For these crystal structures, the strongest accordance between the measured diffraction patterns of PCN and the corresponding theoretical diffraction patterns can be found. Nevertheless, due to the specific character of the measured diffraction patterns with its broad peaks, we can only draw a qualitative picture. In that picture, we can not explicitely exclude any of the discussed potential forms of PCN. We rather assume the PCN to be a conglomerate of many crystallites with slightly different crystal structures and/or defects, in which buckled and flat, A-A-stacked PCN is the dominant phase. The diameters of the crystallites are approximately $15 \mathrm{~nm}$, estimated with the Scherrer equation on the basis of the reflexes (210) and (001) of flat PCN. ${ }^{28,29}$ We also expect an amorphous content in the PCN mainly resulting in non-specific diffraction intensity between the two major peaks. Furthermore, PCN seems to be the result of several other synthesis routes. ${ }^{30,31}$
We have shown that a triclinic crystal structure $(P 1$ or $P \overline{1})$ with $a=16.2 \AA, b=12.1 \AA, 3.26 \AA \leq c \leq 3.275 \AA$, $\alpha=\beta=90^{\circ}$ and $87^{\circ} \leq \gamma \leq 90^{\circ}$ based on buckled and flat melon sheets can explain all major features of the diffraction patterns of PCN. The strong measured peak at $13.2^{\circ}$ is identified to be a superposition of the reflexes $(\overline{2} 10)$ and $(210)$, the measured peak at $17.9^{\circ}$ is assigned to a superposition of the reflexes $(\overline{3} 10)$ and (310), while the measured peak at $27.2^{\circ}$ can be related to the reflexes (001) or (002). Different buckling and stacking motifs are probable crystallographic deviations that are included within the framework of triclinic PCN. An increasing process temperature leads to a more dense packing along the c-axis and also to a distortion of the melon sheets. The unit cell angle $\gamma$ is reduced to a value of about $87^{\circ}$, leading to non-overlapping $(\overline{2} 10),(210)$ and $(\overline{3} 10),(310)$ reflexes for high process temperatures, and thus to a broadening of the respective peaks in the measured XRD patterns. We have assumed a hexagonal unit cell $P \overline{6}(174)$ for a heptazine-based $g-\mathrm{C}_{3} \mathrm{~N}_{4}$ and illustrated that the heptazine-based as well as the triazine-based g$\mathrm{C}_{3} \mathrm{~N}_{4}$ can only be a minor synthesis outcome of the thermal polycondensation of dicyandiamide or related reactants.

\section{ACKNOWLEDGMENTS}

The authors would like to thank F. Zamponi, M. Wörner and T. Elsässer from the Max-Born-Institute für Nichtlineare Optik und Kurzzeitspektroskopie for professional advices and the oppurtunity to finish this work. Financial support by the German Bundesministerium für Bildung und Forschung (excellence cluster project "Light2Hydrogen", grant No. 03IS2071F) is gratefully acknowledged.

${ }^{1}$ X. C. Wang, K. Maeda, A. Thomas, K. Takanabe, G. Xin, J. M. Carlsson, K. Domen, and M. Antonietti, Nature Materials 8, 76 (2009).

${ }^{2}$ F. Yang, M. Lublow, S. Orthmann, C. Merschjann, T. Tyborski, M. Rusu, S. Kubala, A. Thomas, R. Arrigo, M. Hävecker, and T. Schedel-Niedrig, ChemSusChem 5, 1227 (2012).

${ }^{3}$ F. Yang, V. Kuznietsov, M. Lublow, C. Merschjann, A. Steigert, J. Klaer, A. Thomas, and T. Schedel-Niedrig, J. Mater. Chem. A 1 (2013).

${ }^{4}$ A. Thomas, A. Fischer, F. Goettmann, M. Antonietti, J.-O. Müller, R. Schlögl, and J. M. Carlsson, J. Mater. Chem. 18, 4893 (2008).

${ }^{5}$ T. Tyborski, C. Merschjann, S. Orthmann, F. Yang, M.-C. LuxSteiner, and T. Schedel-Niedrig, J. Phys.: Condens. Matter 24, 162201 (2012).

${ }^{6}$ C. Merschjann, T. Tyborski, S. Orthmann, F. Yang, K. Schwarzburg, M. Lublow, M.-C. Lux-Steiner, and T. SchedelNiedrig, Phys. Rev. B 87, 205204 (2013).

${ }^{7}$ Y. Wang, X. Wang, and M. Antonietti, Angew. Chem. Int. Ed. 51, 68 (2012).

${ }^{8}$ B. V. Lotsch and W. Schnick, Chem. Eur. J. 13, 4956 (2007).

${ }^{9}$ B. Jürgens, E. Irran, J. Senker, P. Kroll, H. Müller, and W. Schnick, J. Am. Chem. Soc. 125, 10288 (2003).

${ }^{10}$ B. V. Lotsch, M. Döblinger, J. Sehnert, L. Seyfarth, J. Senker, O. Oeckler, and W. Schnick, Chem. Eur. J. 13, 4969 (2007).

${ }^{11}$ A. Sattler and W. Schnick, Z. Anorg. Allg. Chem. 632 (2006). 
${ }^{12}$ D. M. Teter and R. J. Hemley, Science 271, 53 (1996).

${ }^{13}$ E. Kroke and M. Schwarz, Coord. Chem. Rev. 248, 493 (2004).

${ }^{14}$ H. M. Rietveld, J. Appl. Crsyt. 2, 65 (1969).

${ }^{15}$ H. M. Rietveld, Acta Cryst. 22, 151 (1967).

${ }^{16}$ W. I. F. David, K. Shankland, L. B. McCusker, and C. Baerlocher, Structure Determination from Powder Diffraction Data (OXFORD SCIENCE Publications, 2002).

${ }^{17} \mathrm{H}$. Putz and K. Brandenburg, Crystal Impact, Kreuzherrenstr. 102, 53227 Bonn, Germany, http://www.crystalimpact.com/diamond.

${ }^{18}$ G. Bergerhoff, M. Berndt, and K. Brandenburg, J. Res. Natl. Inst. Stand. Technol. 101, 221 (1996).

${ }^{19}$ W. T. Pennington, J. Appl. Cryst. 32, 1028 (1999).

${ }^{20}$ W. Kraus and G. Nolze, Federal Institute for Materials Research and Testing, Richard-Willstätter-Str. 11, 12489 Berlin, Germany.

${ }^{21}$ M. J. Bojdys, J.-O. Müller, M. Antonietti, and A. Thomas, Chem. Eur. J. 14, 8177 (2008).
${ }^{22} \mathrm{~W}$. Rasband, ImageJ, U. S. National Institutes of Health, Bethesda, Maryland, USA, http://imagej.nih.gov/ij/ (19972012).

${ }^{23}$ M. Abramoff, P. Magalhaes, and S. Ram, Biophotonics International 11, 36 (2004).

${ }^{24}$ J. Sehnert, K. Baerwinkel, and J. Senker, J. Phys. Chem. B 111, 10671 (2007).

${ }^{25}$ P. Coppens, X-Ray Charge Densities and Chemical Bonding (Oxford University Press, Inc., 1997).

${ }^{26}$ G. Vidal-Valat and J.-P. Vidal, Acta Cryst. A 48, 46 (1992).

${ }^{27}$ L. Seyfarth, J. Seyfarth, B. V. Lotsch, W. Schnick, and J. Senker, Phys. Chem. Chem. Phys. 12, 2227 (2010).

${ }^{28}$ P. Scherrer, Göttinger Nachrichten 2, 98 (1918).

${ }^{29} \mathrm{M}$. Birkholz, Thin film analysis by X-ray scattering (Wiley-VCH, 2005).

${ }^{30}$ J. Liu, T. Zhang, Z. Wang, G. Dawson, and W. Chen, J. Mater. Chem. 21, 14398 (2011).

${ }^{31}$ F. Dong, Y. Sun, L. Wu, M. Fu, and Z. Wu, Catal. Sci. Technol. 2, 1332 (2012). 